\title{
Coastline Extraction on Remote Sensing Image Using Gaussian Process Classification
}

\author{
Xiaochuan $\mathrm{Hu}^{1, \mathrm{a}}$, Zihua Qin ${ }^{1, \mathrm{~b}}$, Jinhuan Wang ${ }^{1, \mathrm{c}}$ and Guoshao $\mathrm{Su}^{1, \mathrm{~d}}$ \\ ${ }^{1}$ School of Civil Engineering and Architecture, Guangxi University, Nanning, Guangxi, China 530004 \\ ah_xchuan@163.com, binzihuaqfx@163.com, cwangjinhuan99@163.com, dsuguoshao@163.com
}

Keywords: Gaussian process classification, remote sensing image, coastline extraction.

Abstract. For more rapid and accurate coastline extraction methods, this paper presents a method of coastline extraction on remote sensing image using Gaussian process classification and then the coastline in coastal waters of Beihai city in Guangxi has been extracted. The results show that coastline extraction on remote sensing image using Gaussian process classification overcomes a series of public problems about coastline extraction that over-learning and the limitations of poor generalization ability in small sample exist in Artificial Neural Network and hyper-parameters are difficult to choose for Support Vector Machine. At last, the success of coastline extraction provides a efficient computing method for measurement and identification of coastline.

\section{Introduction}

Coastline is one of the most important linear features on the earth's surface, which displays a dynamic nature [1]. At the same time, it is also the datum line of distinguishing management area between marine and land, the significant contents of Human studies that the interaction between the marine and land, influence of Human activities on the coastal zone and offshore ecosystems. The changes of the coastline change the beach resources and environment of intertidal zone and affect the survival and development of the people directly. And therefore, fast and accurate coastline extraction is of great significance.

Remote sensing has strong abilities of data acquisition, observation for different scales of space and time information and overcomes the disadvantages of traditional coastline extraction methods that measures coastline artificially with GPS which is time-consuming, of low efficiency and accuracy. With the continuous development of remote sensing technology, increasing resolution of remote sensing image and unceasing improvements of the artificial intelligence science in recent years, methods of coastline extraction based on Artificial Neural Network (ANN) and Support Vector Machine (SVM) are coming and many achievements were obtained [2-3]. However, these methods possess a serious of public shortcomings that over-learning and the limitations of poor generalization ability in small sample exist in ANN and hyper-parameters are difficult to choose for SVM. So, looking for a new fast and accurate method of coastline extraction based on remote sensing image is of great importance.

Gaussian Process (GP) is a new machine learning method developed in recent years, has the strict basis of statistical learning theory and also a good adaptability for dealing with complex problems, such as nonlinear, high dimension and small samples [4]. Moreover, GP is a nuclear learning machine which has the characteristic of probability significance, and also able to make a explanation of the probability. Now, it has become a hot research topic in the field of machine learning, and has been successfully used in many fields [5-6]. Therefore, the method of coastline extraction on remote sensing image using Gaussian process classification (GPC) is worth studying.

\section{The Basic Principle of Coastline Extraction Using GPC}

GP for binary classification is described as follows. Let denote the class label corresponding to an input $x$. The probability of success $p(y=1 \mid x)$ is related to an unconstrained latent function $f(x)$.

In the GPC model Bayesian inference is performed about the latent function in the light of observed data. The prior distribution of latent function is: 


$$
p(f \mid \boldsymbol{X}, \theta)=N(0, \boldsymbol{K})
$$

The covariance matrix is defined element-wise, $K_{i j}=k\left(x_{i}, x_{j}, \theta\right) . \theta$ is a set of hyper-parameters. Covariance function can be selected based on the law of true data in GP, but it must meet the conditions that the covariance function can create a nonnegative definite covariance matrix for every point sampling. The commonly used covariance function is:

$$
k_{y}\left(x_{p}, x_{q}\right)=\sigma_{f}^{2} \exp \left(-\frac{1}{2 l^{2}}\left(x_{p}-x_{q}\right)^{2}\right)
$$

The hyper-parameters, $\sigma_{f}$ and 1 , can be achieved by maximum likelihood method. Namely, it can be achieved through the establishment of the logarithmic likelihood function of conditional probability in the training sample and then, partial differential of hyper-parameters was implemented. At last, the optimal solution of hyper-parameters can be sought out by using conjugate gradient optimization method. The logarithmic likelihood function is:

$$
L=\log p(\boldsymbol{y} \mid \boldsymbol{X})=-\frac{1}{2} \boldsymbol{y}^{T}(\boldsymbol{K})^{-1} \boldsymbol{y}-\frac{1}{2} \log |\boldsymbol{K}|-\frac{n}{2} \log 2 \pi
$$

After obtaining the optimal parameters according to Eq. (2), the output value of maximum probability with corresponding to $\boldsymbol{X}_{*}$ can be predicted based on Bayesian inference. The Bayesian inference updates probability distribution constantly by using the observed data. At last, the approximate posterior distribution of $\boldsymbol{y}_{*}$ can be obtained in case of practice sample, $\left(\boldsymbol{x}_{i}, y_{i}\right)$, and forecast sample, $\boldsymbol{X} *$. The approximate posterior distribution of $y *$ is:

$$
p\left(\boldsymbol{y}_{*} \mid \boldsymbol{y}, \boldsymbol{X}, \boldsymbol{X}_{*}\right)=N\left(\boldsymbol{y}_{*} ; \mu_{*}, \sigma_{*}^{2}\right)
$$

where mean and variance are:

$$
\begin{aligned}
& \mu_{*}=\boldsymbol{K}^{\mathrm{T}}\left(\boldsymbol{X}^{*}\right)-\boldsymbol{K}^{-1} \boldsymbol{y} \\
& \sigma_{*}^{2}=\boldsymbol{K}\left(\boldsymbol{X}_{*}, \boldsymbol{X}_{*}\right)-\boldsymbol{K}^{\mathrm{T}}\left(\boldsymbol{X}_{*}\right)(\boldsymbol{K})^{-1} \boldsymbol{K}\left(\boldsymbol{X}_{*}\right)
\end{aligned}
$$

The main purpose of classification models is to predict the class label $y^{*}$ for test inputs $\boldsymbol{X}_{*}$.

$$
\left\{\begin{array}{l}
p\left(y_{*}=+1 \mid \boldsymbol{X}, \boldsymbol{y}, \boldsymbol{x}_{*}\right)=\Phi\left(\frac{\mu_{*}}{\sqrt{1+\sigma_{*}^{2}}}\right) \\
\text { if } p \geq 0.5, y_{*}=+1, \text { otherwise, } y_{*}=-1
\end{array}\right.
$$

where the $\Phi$ is cumulative probability density function of standard normal distribution. Of course, it can be substituted by other alternative response function.

\section{Application Example}

TM image, sensor is TM and orbital is 125/045, in coastal waters of Beihai city in Guangxi was used for band combination, geometric correction, clipping and other pretreatments. TM image was get on November 17, 2001 and its spatial resolution is thirty meters. It is worth mentioning that the fourth band, near infrared band, and the third band, red band, and the second band, green band were used for band combination. And then, standard false color image for coastline extraction was obtained. At last, remote sensing image that contains coastline information was acquired. The remote sensing image shows in Fig 1.

Procedures of Coastline Extraction Using GPC. Procedures are described as follows:

Step 1. The initial sensing image, Fig 1, contains many complex factors affecting coastline extraction. So, calculating the normalized difference water index (NDWI) of the image is necessary [7].Then, the image calculated with NDWI can be obtained and is shown in Fig 2. The NDWI is:

$N D W I=\left(\rho_{\text {Green }}-\rho_{\text {NIR }}\right) /\left(\rho_{\text {Green }}+\rho_{\text {NIR }}\right)$ 
where $\rho_{\text {Green }}$ is green band and $\rho_{\text {NIR }}$ is near infrared band.

Step 2. $N$ sample points, with obvious water features and $N$ sample points, with obvious land features are selected separately in Fig 2 that contains coastline information.

Step 3. The value of R, G, B of sample points $(2 N)$ is extracted and constitute the training sample with its corresponding target value. The training sample is $\left(\boldsymbol{x}_{i}, y_{i}\right), 1 \leq i \leq 2 N$, and $\boldsymbol{x}_{i}=[\mathrm{R}, \mathrm{G}, \mathrm{B}]$, is the input vector of the training sample, $y_{i}$ is corresponding target value. As training sample is water, $y_{i}=-1$, otherwise, $y_{i}=+1$.

Step 4. The value of $\mathrm{R}, \mathrm{G}, \mathrm{B}$ of the remote sensing image is extracted and constitutes the input vector of test sample, $\boldsymbol{X}_{*}$. where $\boldsymbol{X}_{*}=\left[\boldsymbol{x}_{1}, \boldsymbol{x}_{2}, \ldots \boldsymbol{x}_{m}\right]^{\mathrm{T}}, \boldsymbol{m}$ is the number of pixels of remote sensing image.

Step 5. The training sample, $\left(\boldsymbol{x}_{i}, y_{i}\right)$, is trained. And then, the classifier, GPC, which has good generalization ability, is shown in Eq.(7).

Step 6. The output value predicted by Eq.(7), $y^{*}$, can be obtained by inputting the $X_{*}$ into Eq.(7). And then, the segmentation of remote sensing image has been finished and is shown in Fig 3. As $y_{*}=-1$, the pixel is classified as waters. As $y_{*}=+1$, the pixel is classified as land.

Step 7. Sensing segmentation results, shown in Fig 3, divided in above steps is translated into Binary image, Fig 5, after graying and binaryzation, according to grey histogram, Fig 4.

Step 8. The accurate coastline, shown in Fig 6, is obtained by means of vectorization which is based on Fig 5.

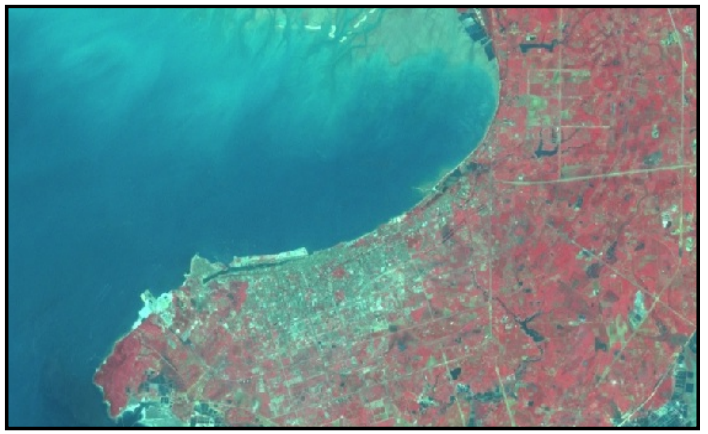

Fig 1 Remote sensing image

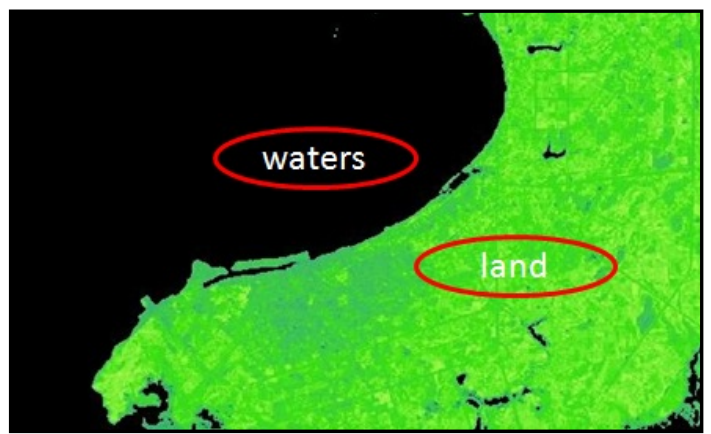

Fig 3 Sensing segmentation results

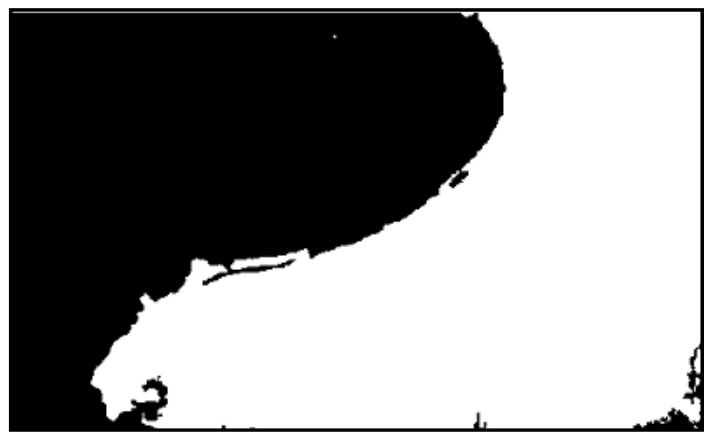

Fig 5 The binary image

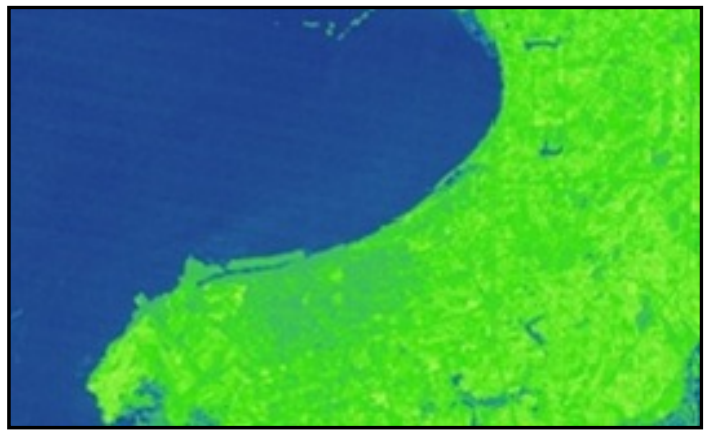

Fig 2 The NDWI

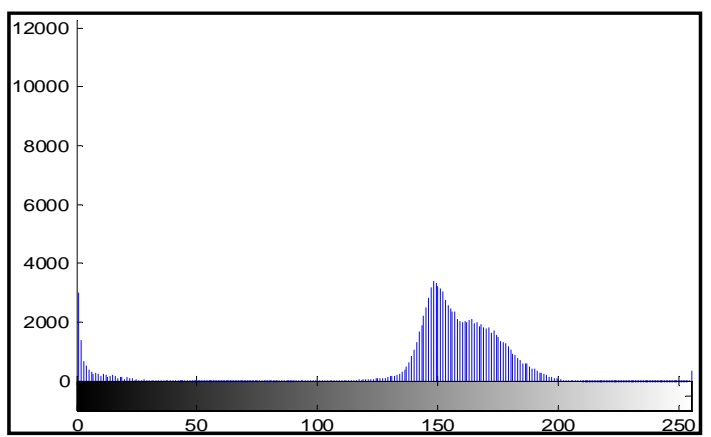

Fig 4 The grey histogram

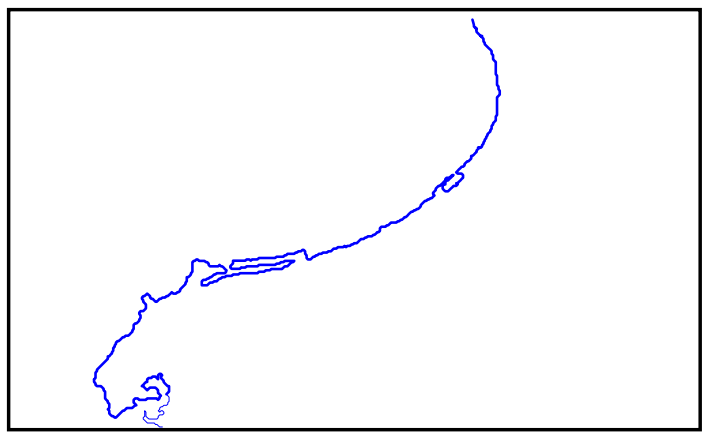

Fig 6 The result of coastline extraction 


\section{The Results Analysis of Coastline Extraction}

The NDWI of water and land has obvious difference. So, the NDWI, shown in Fig 2, can distinguish waters and land effectively. The calculation of NDWI, based on Fig 1, is finished in remote sensing image processing software, ERDAS IMAGINE.

Fig 3 shows that classifier, $\mathrm{Eq}(8)$, identifies the waters and land effectively. Also, the method distinguishes the complex interferences well. And now, the coastline has demonstrated clearly.

Being not able to vectorize color image in MATLAB, graying and binarization on Fig 3 are necessary for high precision coastline. After graying, its grey histogram which is shown in Fig 4 presents a bimodal distribution obviously, so the gray value, 50, is selected as threshold level of the binaryzation. Then, the binary image is acquired and shown in Fig 5. It is worth noting, a reasonable choice of gray threshold is indispensable to get binary image.

Fig 6 is the result of vectorization on binary image for coastal waters of Beihai city in Guangxi. By comparing the Fig 6 and Fig 1, coastline extraction result using GPC matches the lake shoal well. Coastline extraction on remote sensing image using GPC is successful.

\section{Conclusions}

The coastline, not only reliable but also accurate, in coastal waters of Beihai city in Guangxi has been extracted successfully using GPC. The method overcomes a series of existing problems that over-learning and the limitations of poor generalization ability in small sample exist in ANN and hyper-parameters are difficult to choose for SVM in recent years. And also, it provides a good engineering application value for the protection of the coastal landform, development and utilization of tidal flats resources and orderly development of coastal land and resources.

Spectral features are considered merely in this paper, the context information and space characteristics have been ignored. How to use the space characteristics on the basis of making full use of its spectral features to realize the precise positioning of different types of coastline remains to be further study.

\section{Acknowledgements}

This work was financially supported by the Science and Technology Project of the Department of Water Resources of Guangxi Zhuang Autonomous Region (Grant No. 201522).

\section{References}

[1] Ali A Alesheikh, A Ghorbanali, N Nouri: ENVIRON SCI TECHNOL, Vol. 4(2007), p 61-66

[2] Ryan T W, Semintilli P J, Yuen P, PE\&RS Vol. 57(1991), p 947-955

[3] Changming Zhu, Xin Zhang, Jiancheng Luo: Remote Sensing for Land and Resources, Vol. 25(2013), p 69-74 (In Chinese).

[4] Williams C E R A: Gaussian Processes for Machine Learning (MIT Press, Massachusetts 2006)

[5] Mark Girolami, Simon Rogers. Variational: NEURAL COMPUT, Vol. 18(2006), p 1790-1817.

[6] TW Ryan, PJ Sementilli, P Yuen: PE \& RS, Vol. 57(1991), p 947-955.

[7] Bo-Cai Gao: REMOTE SENS ENVIRON, Vol. 58(1996), p 257-266. 